\title{
Ultrasound-Guided Transthoracic Mediastinal Biopsy: A Safe Technique for Tissue Diagnosis in Middle- and Low-Income Countries
}

\author{
Muhammad Kashif Shazlee ${ }^{1}$, Muhammad Ali ${ }^{1}$, Muhammad Saad Ahmed ${ }^{1}$, Junaid Iqbal ${ }^{1}$, Jaideep Darira ${ }^{1}$ \\ , Muhammad Qasim Naeem ${ }^{1}$ \\ 1. Diagnostic Radiology, Dr. Ziauddin Hospital, Karachi, PAK
}

Corresponding author: Muhammad Kashif Shazlee, kashifshazlee@gmail.com

\section{Abstract \\ Background and objectives}

The high cost of video-assisted transthoracic procedures precludes their use in the diagnostics of mediastinal masses in low- and middle-income countries (LMICs). This study aims to assess the technical success rate and diagnostic yield of ultrasound-guided transthoracic mediastinal biopsies at a tertiary care hospital.

\section{Methods}

This descriptive cross-sectional study was conducted in patients presenting with mediastinal masses referred to radiology services at Dr. Ziauddin University Hospital. Karachi, Pakistan. Ultrasonography was performed using Toshiba Xario 200 \& Aplio 500 using convex and linear probes accordingly. Biopsy was performed using a combination of $18 \mathrm{G}$ semiautomatic trucut and $17 \mathrm{G}$ co-axial needles. Complications and overall diagnostic yields were determined.

\section{Results}

In all 70 patients referred, the procedure was completed successfully with an overall procedural yield of $95.7 \%$. Inconclusive biopsies due to inadequate specimen were seen in two (4.2\%) patients. No postprocedure major complication or mortality was observed. Minor complications were seen in three (4.2\%) out of 70 , including hematoma $(<3 \mathrm{~cm})$ in one patient and small pneumomediastinum in two patients.

\section{Conclusion}

Ultrasound-guided transthoracic mediastinal biopsy may be the pragmatic technique of choice in LMICs for the diagnosis of mediastinal masses as they provide real-time visualization and is cost-effective and safe.

Review began $12 / 09 / 2020$ Review ended 02/25/2021 Published 03/16/2021

\section{(๑) Copyright 2021}

Shazlee et al. This is an open access article distributed under the terms of the Creative Commons Attribution License CC-BY 4.0., which permits unrestricted use, distribution, and reproduction in any medium, provided the original author and source are credited.
Categories: Radiology

Keywords: ultrasound guided transthoracic mediastinal biopsy, mediastinal mass, mediastinal tumours, mediastinal biopsy associated mortality, mediastinal biopsy related complications

\section{Introduction}

Anterior mediastinal masses could be a sign of large-spectrum underlying pathologies ranging from benign masses to malignant tumors [1]. Accurate and early diagnosis in this regard can be that fine line that distinguishes complete cure and recovery from mortality. For instance, timely diagnosis of thymic malignancy of advanced stage can be successfully treated with neoadjuvant treatment before surgery [2]. Similarly, lymphomas and germ cell tumors, among other malignancies, can be treated if early diagnosis is made [3-6].

There are numerous other examples that show the importance of early diagnosis and the potential benefits gained from it [7,8]. However, gold standard histopathology-based diagnosis needs tissue sample for which the access to the mediastinal mass is required. There are several methods for obtaining tissue samples for the diagnosis of mediastinal lesions. These include surgical techniques such as video-assisted thoracoscopic biopsy, transbronchial needle biopsy, and endoscopic ultrasound (US)-guided fine-needle aspiration biopsy.

In order to achieve optimal access to the mass, the video-assisted transthoracic surgery (VATS) is deemed superior to other techniques due to adequate visualization despite limited access to the mediastinum. However, VATS cost approximately 1,400 USD per procedure, making this technique unfavorable in a resource-limited country like Pakistan. Therefore, accurate, timely, and cost-effective diagnosis of such masses is of crucial importance. 
In Pakistan, the double burden of communicable and non-communicable diseases has posed an even greater risk to the misdiagnosis, or worse, no diagnosis at all, henceforth grave clinical outcomes in the situation where high-fidelity diagnostic techniques are unaffordable or unavailable to the patients. Our study aims to evaluate the technical outcomes of US-guided transthoracic mediastinal biopsies in patients presenting with mediastinal masses for tissue sampling for histopathology at a tertiary care hospital.

\section{Materials And Methods}

This prospective study was conducted between October 2013 and October 2016 and included 70 consecutively enrolled patients referred to the department of radiology, Dr. Ziauddin University Hospital, Karachi, Pakistan, for histopathological tissue sampling of mediastinal masses or insinuating mediastinal lesions with anterior or posterior compartment involvement, as seen on cross-sectional contrast imaging followed by US-guided transthoracic mediastinal biopsy. The study was conducted following prior approval from the Institutional Ethical Review Committee.

After reviewing the scans, only those patients with adequate acoustic window of the mass were included. Patients with pure cystic lesions or widely necrotic tissue, INR greater than 1.8 , inability to maintain the desired position for the procedure, and pediatric patients with any contraindication to general anesthesia were excluded.

In pediatric patients, the procedure was performed under general anesthesia, whereas local anesthesia was used in adults. Ultrasonography was performed using Toshiba Xario 200 \& Aplio 500 (Toshiba Medical Systems, Tokyo, Japan) using convex and linear probes accordingly. Color Doppler imaging was used in all patients to assess the location of large vessels in close proximity. Co-axial US-guided transthoracic mediastinal biopsy was performed with the needle directed away from the major vessels and heart with a maximum three passes in each patient. All biopsies were taken with a combination of $18 \mathrm{G}$ semiautomatic trucut needle and $17 \mathrm{G}$ co-axial needle. In lesions with necrosis, peripheral soft tissues (enhancing on contrast CT) were targeted. If the parasternal approach was not possible, transsternal (in the pediatric group), suprasternal, or paravertebral approaches were employed.

All patients were kept under observation for the next four hours for monitoring of vitals as per department protocol. A follow-up erect chest radiograph was obtained after one hour in all patients. US Doppler evaluation was repeated prior to discharge. Histological samples were stored in formaldehyde solution and sent for histopathological reporting. These were collected for final diagnoses.

\section{Results}

The mean age of the patients was $32.45 \pm 31$ years (range: 2.5 years to 70 years). Of all the patients, 58 ( $82.8 \%$ ) were males and 38 (53.2\%) were pediatric patients.

The majority of cases had masses within the anterior mediastinum ( $42.8 \%$ [n=30]) followed by the middle mediastinum (35.7\% [n=25]) and posterior mediastinum (14.2\% [n=10]), whereas five (7.1\%) out of 70 patients had mass in the anterior chest wall with mediastinal invasion.

The procedure was successfully completed in all patients (100\% technical success rate). In 68 out of 70 patients, the tissue sample was adequate for diagnostic biopsies, giving an overall procedural yield of $95.7 \%$. An inconclusive biopsy was reported in two (4.2\%) patients due to inadequate tissue specimen, who were then suggested surgical biopsy. No major complication or mortality was observed in the study participants. Minor complications were seen in 3 (4.2\%) out of 70 patients. This included a small hematoma of less than 3 $\mathrm{cm}$ in one patient and small pneumomediastinum in two patients. All minor complications were selflimiting as there were no clinical signs of distress or change in hemodynamics. However, a repeat radiograph was performed at the time of discharge again to confirm any progression.

Malignant masses were seen in $84.2 \%(n=59 / 70)$ on histopathology. Lymphoma was the most common malignancy and seen in $55.9 \%(n=33 / 59)$ of the patients. The most common non-malignant lesion was neurogenic tumors and seen in $63.6 \%(n=7 / 11)$ of the patients.

Figures 1-3 show a large anterior mediastinal mass. US-guided parasternal needle placement was performed for transthoracic mass biopsy. 


\section{Cureus}

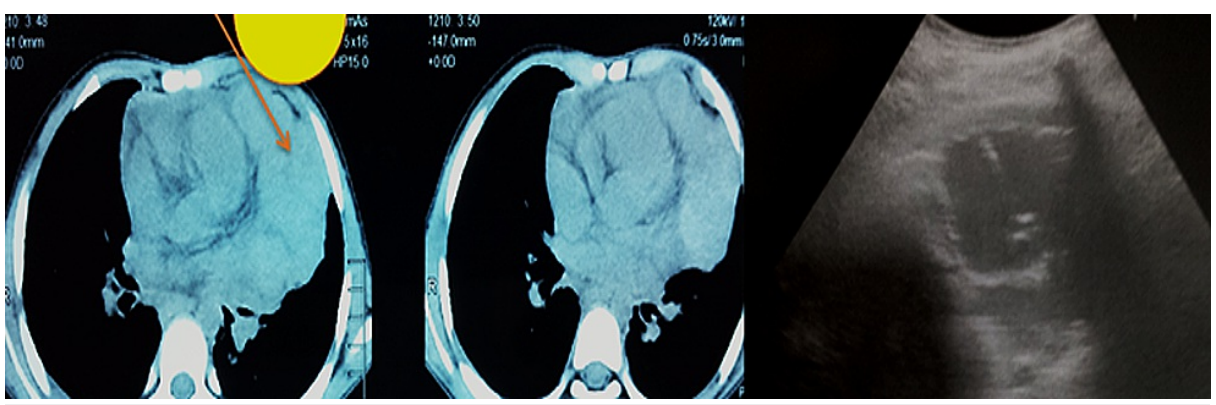

FIGURE 1: Plain CT scan of the chest showing a large anterior mediastinal mass along the pericardium. Ultrasound-guided accurate needle placement was performed for transthoracic mass biopsy.

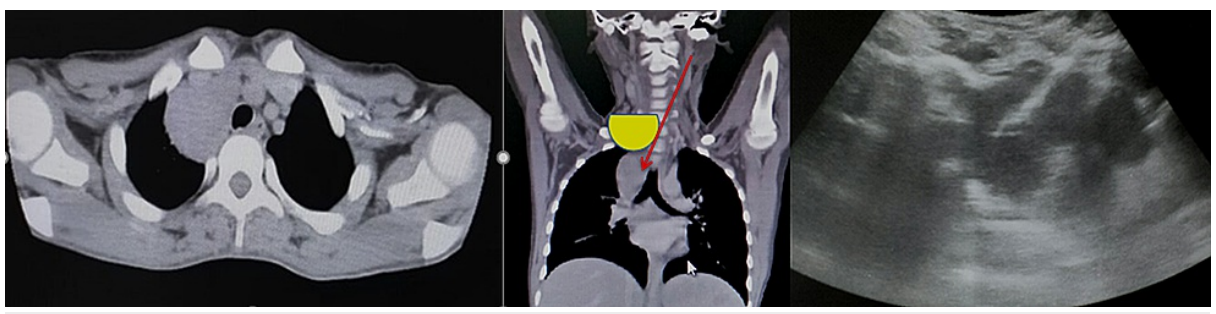

FIGURE 2: Plain CT scan of the chest showing a large anterior mediastinal mass in the right paratracheal location. Ultrasound-guided para-sternal needle placement was performed for transthoracic mass biopsy.

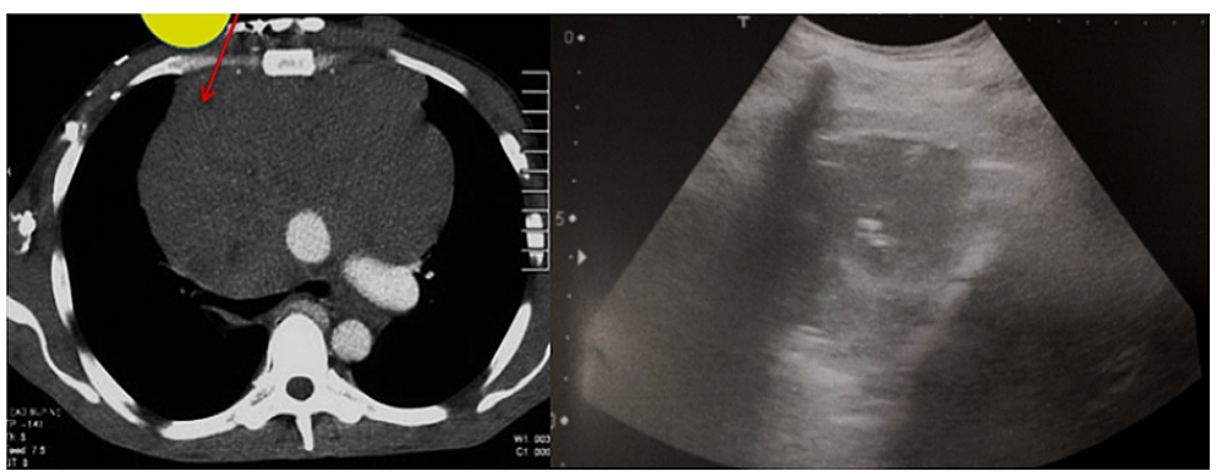

FIGURE 3: Plain CT scan of the chest showing a large anterior mediastinal mass. Ultrasound-guided para-sternal needle placement was performed for transthoracic mass biopsy.

\section{Discussion}

In the recent past, attention has been redirected towards the use of US-guided interventional procedures in terms of cost, enabling real-time visualization and repeatability without radiation hazards. In case of transthoracic mediastinal biopsy, US-guided procedures have proven to be of high accuracy, providing adequate samples for histopathology, without severe complications or highly cost [9]. Cost is of special consideration when it comes to the diagnosis of mediastinal masses in LMICs like Pakistan where the incidence of conditions leading to such masses is on the rise [10]. In our study, the average cost of the procedure was 13,000 PKR (112 USD) at our center compared to approximately 200,000 PKR (1,400 USD) for VATS. Our study findings showed excellent technical success of the procedure comparable to that of VATS.

Primary mediastinal masses account for $3 \%$ of tumors involving the chest wall [11,12]. Around 25-40\% are potentially malignant [11] and are more likely to be located in the anterior mediastinum (Abstract: Eames R, 
Kim Y, How E, Wong L. Anterior mediastinal mass. 2019). The most common lesions encountered in the mediastinum are thymoma, neurogenic tumors, and benign cysts, altogether representing $60 \%$ of patients with mediastinal masses [12]. Correct diagnosis of a mediastinal tumor depends on clinical and radiological history, imaging findings, and histopathological diagnosis.

Of the surgical approaches, VATS is popular because of safety and accuracy as compared to cervical mediastinoscopy [13-15]. Despite this notion, the VATS is expensive procedure for middle-income country like Pakistan and even more impractical for the low-income countries. However, imaging-guided biopsy of anterior mediastinal masses offers less complications as it is less invasiveness [16-18].

\section{Conclusions}

US-guided transthoracic mediastinal biopsy is a pragmatic technique of choice in LMICs for the diagnosis of mediastinal masses as it is cost-effective and safe, and provides real-time visualization.

\section{Additional Information \\ Disclosures}

Human subjects: Consent was obtained or waived by all participants in this study. Department Research Committee, Dr. Ziauddin Hospital issued approval 0130319MARAD. Protocol Title: "Ultrasound guided transthoracic mediastinal biopsy: a safe technique for tissue diagnosis in middle and low income countries" Dear Dr. Muhammad Kashif Shazlee, Thank you for submitting the above mentioned study proposal. The department research committee, Dr. Ziauddin Hospital Karachi has reviewed this project in the meeting of 9th August 2013 and has given approval to conduct this study. Any change in protocol should be notified to the committee for prior approval. Kindly notify us when the research is complete. Animal subjects: All authors have confirmed that this study did not involve animal subjects or tissue. Conflicts of interest: In compliance with the ICMJE uniform disclosure form, all authors declare the following: Payment/services info: All authors have declared that no financial support was received from any organization for the submitted work. Financial relationships: All authors have declared that they have no financial relationships at present or within the previous three years with any organizations that might have an interest in the submitted work. Other relationships: All authors have declared that there are no other relationships or activities that could appear to have influenced the submitted work.

\section{References}

1. Halpenny D, Niu B, McGuinness G, Bessich J, Berman P, Lowy J, Ko J: Incidentally detected mediastinal mass on a chest radiograph. Ann Am Thorac Soc. 2017, 14:459-462. 10.1513/AnnalsATS.201603-162CC

2. Korst RJ, Bezjak A, Blackmon S, et al.: Neoadjuvant chemoradiotherapy for locally advanced thymic tumors: a phase II, multi-institutional clinical trial. J Thorac Cardiovasc Surg. 2014, 147:36-46. 10.1016/j.jtcvs.2013.08.061

3. Klein JS, Salomon G, Stewart EA: Transthoracic needle biopsy with a coaxially placed 20-gauge automated cutting needle: results in 122 patients. Radiology. 1996, 198:715-720. 10.1148/radiology.198.3.8628859

4. Klein JS, Zarka MA: Transthoracic needle biopsy. Radiol Clin North Am. 2000, 38:235-266.

5. Larscheid RC, Thorpe PE, Scott WJ: Percutaneous transthoracic needle aspiration biopsy: a comprehensive review of its current role in the diagnosis and treatment of lung tumors. Chest. 1998, 114:704-709. 10.1378/chest.114.3.704

6. Westcott JL, Rao N, Colley DP: Transthoracic needle biopsy of small pulmonary nodules . Radiology. 1997, 202:97-103. 10.1148/radiology.202.1.8988197

7. Date H: Diagnostic strategies for mediastinal tumors and cysts. Thorac Surg Clin. 2009, 19:29-35. 10.1016/j.thorsurg.2008.09.001

8. Adler O, Rosenberger A: Invasive radiology in the diagnosis of mediastinal masses. Use of fine needle for aspiration biopsy. Der Radiologe. 1979, 19:169-172.

9. Richardson CM, Pointon KS, Manhire AR, Macfarlane JT: Percutaneous lung biopsies: a survey of UK practice based on 5444 biopsies. Br J Radiol. 2002, 75:731-735. 10.1259/bjr.75.897.750731

10. Karki S, Chalise S: Analysis of mediastinal lesions: a study of 27 cases . J Pathol Nepal. 2011, 2:114-117. 10.3126/jpn.v1i2.5404

11. Vaziri M, Pazooki A, Zahedi-Shoolami L: Mediastinal masses: review of 105 cases . Acta Medica Iranica. 2009, 297:300.

12. Juanpere S, Cañete N, Ortuño P, Martínez S, Sanchez G, Bernado L: A diagnostic approach to the mediastinal masses. Insights Imaging. 2013, 4:29-52. 10.1007/s13244-012-0201-0

13. Larsen SS, Krasnik M, Vilmann P, Jacobsen GK, Pedersen JH, Faurschou P, Folke K: Endoscopic ultrasound guided biopsy of mediastinal lesions has a major impact on patient management. Thorax. 2002, 57:98-103. 10.1136/thorax.57.2.98

14. Dixit R, Shah NS, Goyal M, et al.: Diagnostic evaluation of mediastinal lesions: analysis of 144 cases . Lung India. 2017, 34:341-348. 10.4103/lungindia.lungindia_311_16

15. De Leyn P, Dooms C, Kuzdzal J, et al.: Revised ESTS guidelines for preoperative mediastinal lymph node staging for non-small-cell lung cancer. Eur J Cardiothorac Surg. 2014, 45:787-798. 10.1093/ejcts/ezu028

16. Trejo Bittar HE, Doberer D, Mehrad M, Strollo DC, Leader JK, Wenzel S, Yousem SA: Histologic findings of severe/therapy-resistant asthma from video-assisted thoracoscopic surgery biopsies. Am J Surg Pathol 2017, 41:182-188. 10.1097/PAS.0000000000000777

17. Irons JF, Miles LF, Joshi KR, Klein AA, Scarci M, Solli P, Martinez G: Intubated versus nonintubated general 


\section{Cureus}

anesthesia for video-assisted thoracoscopic surgery-a case-control study. J Cardiothorac Vasc Anesth. 2017, 31:411-417. 10.1053/j.jvca.2016.07.003

18. Donnell C, Nadarjan P, Doolan N, et al.: Video-assisted thoracoscopic surgical (VATs) biopsy in interstitial lung disease-experience in 3 Irish centres. Eur Respiratory Soc. 2017, 50:2452. 10.1183/1393003.congress2017.PA2452 